\title{
Spectrum of Multisystem Inflammatory Syndrome in Children (MIS-C)—a Report of Three Cases
}

\author{
Shruti Jain ${ }^{1} \cdot$ Archana Kashyap $^{1} \cdot$ Amitabh Singh $^{1}$ (I) \\ Accepted: 1 September 2021 / Published online: 21 September 2021 \\ (c) The Author(s), under exclusive licence to Springer Nature Switzerland AG 2021
}

\begin{abstract}
We report three cases of multisystem inflammatory syndrome in children (MIS-C) during July 2020 from a tertiary care hospital with different clinical presentations and course of management. This will guide in better management of children with MIS-C. All three patients, aged 1 to 12 years old, were critically ill. They presented with common features of MIS-C, such as fever, conjunctival congestion, gastrointestinal involvement, and skin manifestations. Clinical features were suggestive of shock, coagulopathy, and multiorgan involvement. Laboratory findings revealed raised inflammatory markers, including C-reactive protein (CRP), erythrocyte sedimentation rate (ESR), and D-dimers (DD). All patients required intensive care with oxygen therapy, fluid resuscitation, inotropic agents, and broad-spectrum antibiotics. All patients received steroids, and two patients were given intravenous immunoglobulin. One patient died, and the remaining two patients were discharged. Our findings confirmed that COVID-19 may cause severe disease in children, and the presentation may vary, requiring early recognition and timely management.
\end{abstract}

Keywords COVID-19 $\cdot$ Multisystem inflammatory disease in children $\cdot$ Methyl prednisolone $\cdot$ Shock $\cdot$ Toxic shock syndrome

\section{Introduction}

Coronavirus disease 2019 (COVID-19) is caused by the severe acute respiratory syndrome coronavirus 2 (SARSCoV-2). The COVID-19 pandemic, which was declared in early 2020, has infected the world's population, including adults and children. MIS-C is a rare and serious complication of COVID-19, and management guidelines are evolving. We share our experience of management of these patients and outcome which will help in better management of such patients. Various clinical manifestations of the disease have been reported, which are not always consistent. The variations are even greater and atypical in children, which makes diagnosis more difficult. The condition generally has a milder course in children; however, manifestations in children can progress to severe disease and even death

This article is part of the Topical Collection on COVID-19

Amitabh Singh

doc.amitabh@gmail.com

1 Department of Pediatrics, Vardhman Mahavir Medical College and Safdarjung Hospital, New Delhi 110029, India
[1]. COVID-19 presents with typical fever and a flu-like illness, but it may also include findings in the gastrointestinal tract, skin, and central nervous system, along with cardiac involvement. Most patients have a favourable recovery, but others may experience multisystem inflammatory syndrome in children (MIS-C), making diagnosis and management difficult with a potentially grave outcome. MIS-C is a clinically severe illness that may require hospitalisation. There is no definitive diagnostic test for MIS-C, so clinical parameters and supportive laboratory investigations are the mainstay of diagnosis.

Knowledge of the COVID-19 course in the paediatric population has evolved over time, and the Kawasaki disease-like presentation of COVID-19-related MIS-C is being reported around the world. The condition may present in one of three ways: (1) as a COVID-19 acute infection, during which patients have an asymptomatic course or mild symptoms; (2) as a multisystem hyperinflammatory syndrome; or (3) as a hyperimmune phase during acute COVID-19, immediately after the viremia phase (adult type).

The multisystem hyperinflammatory syndrome associated with COVID-19 is categorised as a Kawasaki-like disease or 
a febrile inflammatory state (FIS) and TSS-like presentation of MIS-C [2].

\section{Case Presentation}

Three critically ill children from a tertiary care hospital in New Delhi presented with a spectrum of MIS-C symptoms associated with COVID-19 infection.

\section{Case 1}

A 3-year 6-month-old female presented with febrile illness for 5 days $\left(101-103^{\circ} \mathrm{F}\right)$ associated with conjunctival congestion and decreased appetite. There was no history of cough, cold, breathlessness, diarrhoea, vomiting, or altered sensorium. On arrival at the emergency department, the patient had tachycardia (HR 144), tachypnoea, skin rash, and hypotension. A diagnosis of a Kawasaki-like illness was made, and the child was started on intravenous immunoglobulin $2 \mathrm{gm} / \mathrm{kg} /$ day for 1 day with dobutamine support. The patient had raised inflammatory markers, and a nasopharyngeal swab was positive for COVID-19 reverse transcriptase polymerase chain reaction (RT-PCR) SARS COV2. Intravenous methylprednisolone pulse therapy $30 \mathrm{mg} / \mathrm{kg} /$ day for 3 days was added on clinical worsening. Recurrence of skin lesions was noticed on day 7 of hospitalisation, after having received oral prednisolone initially at $2 \mathrm{mg} / \mathrm{kg} /$ day for 1 week followed by tapering dose to $1 \mathrm{mg} / \mathrm{kg}$ in next 1 week. The patient responded gradually and was discharged, and there was no coronary involvement at the 4-week follow-up cardiac evaluation.

\section{Case 2}

A 1-year 6-month-old male presented after having a rash for 8 days, noticed first on the face but followed by generalised desquamation, accompanied by loose stools for 7 days (high purge rate) and fever $\left(102^{\circ} \mathrm{F}\right)$ with altered sensorium for 2 days prior to admission. The patient was in shock, was hypoxic on room air ( $\mathrm{SpO} 288 \%)$, and had multiorgan involvement with gastrointestinal bleeding. A differential diagnosis of toxic shock syndrome (TSS)/bacterial sepsis was made, and broad-spectrum antibiotics were started. The patient had multiorgan dysfunction with hypotension, oliguria, and liver dysfunction. The patient tested positive for COVID-19 antigen on nasopharyngeal swab using reverse transcription polymerase chain reaction (RT-PCR). The patient received intravenous dexamethasone $0.15 \mathrm{mg} / \mathrm{kg} 2$ doses in view of hypoxia. The patient required mechanical ventilation with vasopressor but died on day 5 of admission.

\section{Case 3}

An 11-year-old male presented with high-grade fever with generalised body aches, intermittent vomiting, and desquamated rash with conjunctivitis for 7 days. Initially in compensated shock, the patient required inotropic support but improved after intravenous immunoglobulin (IVIG) $2 \mathrm{mg} / \mathrm{kg} / \mathrm{day}$ for 1 day and was continued on aspirin. The patient was started on oral prednisolone $2 \mathrm{mg} / \mathrm{kg} /$ day for 1 week, tapered, and stopped over the next 2 weeks. The patient was discharged and was positive for the COVID-19 IgG antibody. The patient had no coronary involvement at the 4-week follow-up.

Table 1 highlights the symptoms and signs of all three patients.

Table 2 highlights the laboratory workup of all three cases.

\section{Discussion}

The COVID-19 pandemic has affected people of all ages, including children. MIS-C is one of the spectrum of COVID-19 sequelae that may cause serious complications. MIS-C is defined as affecting children 0 to 19 years of age, and it produces a fever that lasts more than 3 days with at least two of the following comorbidities: rash, bilateral non-purulent conjunctivitis, mucocutaneous inflammation, hypotension or shock, features of myocardial dysfunction, pericarditis, valvulitis, coronary abnormalities (echocardiography findings, elevated troponin/NT-proBNP levels), coagulopathy (prolonged PT or PTT, elevated DD). Acute gastrointestinal problems (diarrhoea, vomiting, abdominal pain), elevated inflammation markers (ESR, C-reactive protein, procalcitonin) with no other obvious microbial cause of inflammation, and evidence of COVID-19 (RTPCR, antigen, positive serology) or contact with patients with COVID-19 [2]. The pathophysiology of MIS-C is based on an abnormal immune response to the virus causing clinical features similar to Kawasaki disease and macrophage activation syndrome [3, 4]. Many children with MIS-C have a negative SARS-CoV-2 PCR test but are positive for COVID-19 serology [5-8]. Case 3 patient was PCR-negative with a positive COVID-19 serology. All three cases met the criteria for MIS-C. Case 1 had an acute febrile hyperimmune presentation similar to the adult condition. Case 2 had a TSS-like presentation, and case 3 had a Kawasaki-like presentation.

MIS-C, unlike Kawasaki disease, most commonly presents with cardiovascular symptoms (hypotension or depressed cardiac function) and impaired gastrointestinal 
Table 1 Clinical presentation of three cases with MIS-C

\begin{tabular}{llll}
\hline Symptoms & Case 1 & Case 2 & Case 3 \\
\hline Fever (duration) & Yes (5 days) & Yes (7 days) & Yes (7 days) \\
Rash & Maculopapular & Erythema & Desquamation \\
Bilateral non-purulent conjunctivitis & Yes & Yes & Yes \\
Oral mucosal inflammation & Yes & No & Yes \\
Peripheral cutaneous inflammation signs & Yes & Yes & Yes \\
$\quad$ hands or feet) & & & \\
Pale/mottled skin & Yes & Yes & No \\
Cold hands/feet & Yes & Yes & No \\
Prolonged capillary refill time & No & Yes & No \\
Chest pain & No & No & No \\
Diarrhoea & No & Yes & Yes \\
Vomiting & Yes & Yes & Yes \\
Bleeding from any site & No & Yes & No \\
Seizure & No & Yes & No \\
Hypoxia/oxygen requirement & No & Yes & No \\
Hypotension (age appropriate) & Yes & Yes & Yes \\
Urinary output $<2$ mL/kg/h & No & Yes & No \\
Tachypnoea & Yes & Yes & No \\
Tachycardia & Yes & Yes & Yes \\
\hline
\end{tabular}

Table 2 Laboratory parameters of three cases with MIS-C

\begin{tabular}{|c|c|c|c|}
\hline Parameters & Case 1 & Case 2 & Case 3 \\
\hline Hemoglobin (g/dL) & 13.0 & 7.6 & 10.5 \\
\hline Platelets (per $\mu \mathrm{L}$ ) & $2,82,000$ & 62,000 & $3,94,000$ \\
\hline White blood cell count(per $\mu \mathrm{L})$ & 10,800 & 14,100 & 6,900 \\
\hline Neutrophils (per $\mu \mathrm{L})$ & 7,300 & 8,200 & 3,640 \\
\hline LYMPHOCYTES (per $\mu \mathrm{L})$ & 2,500 & 5,250 & 2,370 \\
\hline Prothrombin time/international normalised ratio & $13 / 1.0$ & $16 / 1.4$ & $13.3 / 1.14$ \\
\hline Activated partial thromboplastin time (seconds) & $34 / 34$ & $45 / 34$ & $31.1 / 34$ \\
\hline Urea/creatinine (mg/dL) & $26 / 0.3$ & $66 / 0.6$ & $11 / 0.4$ \\
\hline Sodium/potassium (mEq/L) & $134 / 4.2$ & $>160 / 3.8$ & $133 / 3.7$ \\
\hline $\begin{array}{l}\text { Serum glutamic oxaloacetic transaminase(SGOT)/serum glutamic } \\
\text { pyruvic transaminase(SGPT) (U/L) }\end{array}$ & $32 / 9$ & $1053 / 445$ & $24 / 16$ \\
\hline Total bilirubin $(\mathrm{mg} / \mathrm{dL})$ & 0.2 & 0.6 & 0.3 \\
\hline N-terminal pro-B-type natriuretic peptide (NT-proBNP) & $134 \mathrm{pg} / \mathrm{mL}(<300)$ & - & - \\
\hline Troponin & Negative & - & Negative \\
\hline COVID-19 reverse transcriptase polymerase chain reaction & Positive & Positive & Negative \\
\hline SARS COV2 Ig G ELISA & & & Positive \\
\hline Rapid malarial antigen test & Negative & Negative & Negative \\
\hline Serum Widal & Negative & Negative & Negative \\
\hline Dengue NS1 antigen & Negative & Negative & Negative \\
\hline Urine routine and microbiology & No abnormality & No abnormality & No abnormality \\
\hline Chest radiograph & Normal & Bilateral infiltrates & Normal \\
\hline Blood culture & No growth & contaminants & No growth \\
\hline
\end{tabular}

function [5]. COVID-19 was initially thought to cause severe pneumonia progressing to acute respiratory distress syndrome (ARDS). MIS-C has now been shown to have variable presentations that resemble incomplete Kawasaki disease or TSS [3]. Myocardial dysregulation can be isolated or caused by a combination of factors like ischaemic 
or hypoxic cardiomyopathy, acute viral myocardial injury, or systemic inflammation. It can even be caused infrequently by coronary artery ischaemia [9].

Clinical features similar to those in our cases have been reported across the world. All three of our cases had a fever that lasted 3 to 5 days, which is seen in $100 \%$ of reported cases. GI symptoms in the form of abdominal pain, diarrhoea, and vomiting have been reported in $60-100 \%$ of MIS-C patients and were seen in two of our cases [10-12]. Skin manifestations, which have been seen in $45-76 \%$ of reported paediatric COVID-19 cases in the form of vesicular eruptions, pseudo-chilblains, erythema multiforme-like lesions, palmar erythema, and erythematous rash were also observed in all three of our cases [13-16]. Eye involvement with non-purulent conjunctivitis has been reported in $30-81 \%$ of patients, and all our patients had eye involvement $[5,7,8]$. Mucosal involvement (seen in $27-76 \%$ of reported cases) was seen in only two of our cases with oral ulcerations, whereas respiratory involvement (reported in $21-65 \%$ of cases with features like tachypnoea, difficulty in respiration, and severe pulmonary involvement in the form of ARDS) was seen in only one of our cases. Case 2, which had the most severe presentation, demonstrated neurological involvement with seizures. In other studies, patients have reported neurological components such as headache, lethargy, encephalopathy, seizures, coma, meningoencephalitis, and brain stem and/or cerebellar signs [5, 17]. Other, less commonly reported symptoms in the paediatric population (up to $16 \%$ ) are sore throat, muscle pain/myalgias, limb oedema (both hands and feet), and lymphadenopathy.

Common clinical findings of MIS-C are hypotension or shock (reported in $32-76 \%$ of patients) [5, 8, 11]. Our first case developed hypotension on day 2 of admission, whereas cases 2 and 3 demonstrated hypotension on day 1 of admission, which indicates the severity of the TSSand Kawasaki-like disease elements of COVID-19-related MIS-C. Therefore, rapid identification and management are essential in cases of suspected TSS- or Kawasaki-like illness when COVID-19 is a possible underlying illness.

Laboratory results showed abnormal blood cell counts with lymphocytopenia, neutrophilia, or mild anaemia and thrombocytopenia $[3,5,6,8,11]$. We noted that cases 1 and 3 had normal haemograms, but case 2 showed moderate anaemia, neutrophilia, and thrombocytopenia. This anaemia could be due to underlying illness or bleeding because of disseminated intravascular coagulation (DIC).

The inflammatory profile showed all three cases to be CRP-positive, which has also has been observed in 90-100\% of cases reported in other studies. The markers showed correlation with the severity of illness as well. In cases 1 and 3 , for which almost all markers were tested, the values were confirmatory of the patients' clinical conditions. Interleukin 6 (IL-6), DD, and lactate dehydrogenase (LDH) were comparatively low in case 3 but were significantly high in cases 1 and 2. In patients suspected to have MIS-C, a high CRP value with neutrophilia, thrombocytopenia, and highly deranged kidney function or liver function tests should be evaluated with both serology and RT-PCR on a nasopharyngeal swab. Up to $60 \%$ of patients with a negative PCR have had a positive serology test, and 30-35\% of patients with a negative PCR have had both serology and RT-PCR return positive [5-7, 11]. A large series from India has highlighted high incidence of hospitalised children with COVID-19 infection meeting MIS-C criteria [18].

As more evidence emerge, intravenous immunoglobulin and steroid are being used for critical multisystem inflammatory syndrome in children (MIS-C) with shock or multiorgan dysfunction or Kawasaki phenotype. Steroids are indicated in a child with MIS-C without shock.

The patient with the most severe presentation was case 2 , and the initial presentation was multiorgan dysfunction along with immune dysregulation; hence, the management plan could have included antivirals like Remdesivir or immunomodulators like IVIG, which may have decreased the disease severity.

Multi country multi-site randomised controlled trials are required for better understanding of pathogenesis and management of MIS-C.

\section{Conclusion}

COVID-19 infection in children is mostly mild, although severe complications, such as MIS-C, have been reported. The diagnosis of this complication is primarily based on clinical signs and symptoms, along with supportive investigation. Early diagnosis will assist prompt initiation of management, which will result in better outcomes.

Author Contribution SJ, AK, and AS participated in conception of study, analysis, and interpretation of the data, drafting the article, or revising it critically for important intellectual content and all authors approved the final version.

Data Availability Reported data available for review.

Code Availability Not applicable.

\section{Declarations}

Ethics Approval Obtained from institute ethics committee IEC/VMMC/ SJH/2020-08/CC-42.

Consent to Participate Obtained from legal guardian.

Consent for Publication Obtained from legal guardian. 
Conflict of Interest The authors declare no competing interests.

\section{References}

1. Panahi L, Amiri M, Pouy S. Clinical characteristics of COVID-19 infection in newborns and pediatrics: a systematic review. Arch Acad Emerg Med. 2020;8:e50. Accessed 27 Feb 2021: https:// pubmed.ncbi.nlm.nih.gov/32440661/.

2. World Health Organisation: Multisystem inflammatory syndrome in children and adolescents with COVID-19: scientific brief. (2020). Accessed $27 \mathrm{Feb} 2021$ : https://apps.who.int/iris/bitstream/ handle/10665/332095/WHO-2019-nCoV-Sci_Brief-Multisystem_ Syndrome_Children-2020.1-eng.pdf.

3. Riphagen S, Gomez X, Gonzalez-Martinez C, et al. Hyperinflammatory shock in children during COVID-19 pandemic. Lancet. 2020;395:1607-8. https://doi.org/10.1016/S0140-6736(20) 31094-1.

4. Carter MJ, Fish M, Jennings A, et al. Peripheral immunophenotypes in children with multisystem inflammatory syndrome associated with SARS-CoV-2 infection. Nat Med. 2020;26:1701-7. https://doi.org/10.1038/s41591-020-1054-6.

5. Feldstein LR, Rose EB, Horwitz SM, et al. Multisystem inflammatory syndrome in U.S. children and adolescents. N Engl J Med. 2020;383:334-46. https://doi.org/10.1056/NEJMoa2021680.

6. Verdoni L, Mazza A, Gervasoni A, et al. An outbreak of severe Kawasaki-like disease at the Italian epicentre of the SARS-CoV-2 epidemic: an observational cohort study. Lancet. 2020;395:17718. https://doi.org/10.1016/S0140-6736(20)31103-X.

7. Whittaker E, Bamford A, Kenny J, et al. Clinical characteristics of 58 children with a pediatric inflammatory multisystem syndrome temporally associated with SARS-CoV-2. JAMA. 2020;324:25969. https://doi.org/10.1001/jama.2020.10369.

8. Godfred-Cato S, Bryant B, Leung J, et al. COVID-19-associated multisystem inflammatory syndrome in children-United States, March-July 2020. MMWR Morb Mortal Wkly Rep.

9. Sperotto F, Friedman KG, Son MBF, et al. Cardiac manifestations in SARS-CoV-2-associated multisystem inflammatory syndrome in children: a comprehensive review and proposed clinical approach. Eur J Pediatr. 2021;180:307-22. https://doi.org/10. 1007/s00431-020-03766-6.
10. Webb K, Abraham DR, Faleye A, et al. Multisystem inflammatory syndrome in children in South Africa. Lancet Child Adolesc Health. 2020;4:e38. https://doi.org/10.1016/S2352-4642(20) 30272-8.

11. Belhadjer Z, Méot M, Bajolle F, et al. Acute heart failure in multisystem inflammatory syndrome in children in the context of global SARS-CoV-2 pandemic. Circulation. 2020;142:429-36. https:// doi.org/10.1161/CIRCULATIONAHA.120.048360.

12. Tullie L, Ford K, Bisharat M, et al. Gastrointestinal features in children with COVID-19: an observation of varied presentation in eight children. Lancet Child Adolesc Health. 2020;4:e19-20. https://doi.org/10.1016/S2352-4642(20)30165-6.

13. Recalcati S. Cutaneous manifestations in COVID-19: a first perspective. J Eur Acad Dermatol Venereol. 2020;34:e212-3. https:// doi.org/10.1111/jdv.16387.

14. Galván CC, Català $\mathrm{A}$, Carretero $\mathrm{HG}$, et al. Classification of the cutaneous manifestations of COVID-19: a rapid prospective nationwide consensus study in Spain with 375 cases. Br J Dermatol. 2020;183:71-7. https://doi.org/10.1111/bjd.19163.

15. Estébanez A, Pérez-Santiago L, Silva E, et al. Cutaneous manifestations in COVID-19: a new contribution. J Eur Acad Dermatol Venereol. 2020;34:e250-1. https://doi.org/10.1111/jdv.16474.

16. Landa N, Mendieta-Eckert M, Fonda-Pascual P, et al. Chilblainlike lesions on feet and hands during the COVID-19 pandemic. Int J Dermatol. 2020;59:739-43. https://doi.org/10.1111/ijd.14937.

17 Henry D, Ackerman M, Sancelme E, et al. Urticarial eruption in COVID-19 infection. J Eur Acad Dermatol Venereol. 2020;34:e244-5. https://doi.org/10.1111/jdv.16472.

18. Gupta Dch S, Chopra MdN, Singh MdA, Gera R, Chellani MdH, Pandey PhDR, AroraMd BS. Unusual clinical manifestations and outcome of multisystem inflammatory syndrome in children (MIS-C) in a tertiary care hospital of North India. J Trop Pediatr. 2021;67(1):fmaa127.

Publisher's Note Springer Nature remains neutral with regard to jurisdictional claims in published maps and institutional affiliations. 